\title{
Mild Traumatic Brain Injury - Case Report
}

\begin{abstract}
A mild traumatic brain injury or a concussion represents the majority of all traumatic brain injuries. The consequences show on physical, cognitive, and emotional functioning and even though the injury classifies as mild, it can have a significant effect on a patient, patient's family and their quality of life. Defects are often overlooked as objective clinical methods are lacking. Neuropsychological evaluation can aid in appraisal of the defect magnitude and determine factors that influence the outcome of the injured. The following case report addresses the importance of neuropsychological evaluation in treating cognitive defects along with the Cognitive Behavioral therapy approach toward emotional and behavioral disorders treatment in mild traumatic brain injury. It has been shown how important it is to find possible causes for slow recovery. The annuity tendencies have been noted as an important factor for prolongation of the post-concussion syndrome. We can detect the symptom simulation with appropriate psychological instruments. Described is a case of 38-year-old man who suffered a mild traumatic brain injury.
\end{abstract}

Keywords: Mild traumatic brain injury; Neuropsychology; Neuropsychological assessment; Cognitive re-habilitation; Cognitive behavioral therapy
Volume 3 Issue I - 2015

\author{
Ursa Cizman Staba,' Karmen Resnik ${ }^{2}$ \\ 'Traumatic Brain Injury rehabilitation department, University \\ Rehabilitation Institute Soca, Slovenia \\ ${ }^{2}$ Private employed, Slovenia
}

Correspondence: Ursa Cizman Staba, Principal Investigator, University Rehabilitation Center Soca, Linhartova cesta 5I, 1000 Ljubljana, Slovenia, Tel (0I) 475 8I 00: Email ursa.cizman@ir-rs.si

Received: March 30, 2015 | Published: June 0I, 2015
Abbreviations: TBI: Traumatic Brain Injury; PCS: PostConcussion Syndrome; GCS: Glasgow Coma Scale; CBT: CognitiveBehavioral therapy

\section{Introduction}

\section{Basic features of the mild traumatic brain injury}

A mild traumatic brain injury (TBI) is a worldwide health problem as it represents $70-90 \%$ of all registered brain injuries with a prevalence of $100-300$ per 100.000 or even 600 per 100.000 people. ${ }^{1}$ The mild TBI, also referred to as "concussion", "mild head injury", "mild brain injury" is one of the most prevailing diagnoses among nervous system dysfunctions ${ }^{3,4}$ with a significant portion of the injured who suffer long-term consequences. The most prevalent symptoms soon after the injury are a headache, fatigue, dizziness and irritability. Other often present symptoms include light sensitivity and/or sound sensitivity, attention and con-centration difficulties, memory problems, slower information processing, depression and among less likely to occur also a foggy or double vision. The symptoms usually clear after 3 month ${ }^{5-7}$ whereas in some cases insist for a prolonged period ${ }^{8-13} \mathrm{~A}$ mild TBI rehabilitation is relatively swift and the injured return to their normal, pre-injury functioning. However, $25-30 \%$ of patients report symptoms between 3 to 6 months after the injury and in 10-15\% of the cases, the problems persist even longer. ${ }^{14}$ In those cases, we talk about a chronic post-concussion syndrome, which significantly effects patient's life at home, and in social and occupational contexts. ${ }^{15}$ In the latter, it posts significant monetary problems for the individual and society due to frequent absenteeism. In cases where symptoms persist even after 3 months the longitudinal effects of the mild TBI are expected. ${ }^{10}$ Mild TBIs are often under diagnosed as there are no objective clinical diagnostic methods available and patients often feel misunderstood which often enhances the simulation of the symptoms and has a significant impact on the satisfaction and quality of life. The most common causes of mild TBI are car accidents, falls and sport activity injuries. At higher risk for a mild TBI are younger and older people, men, minorities, people with lower socioeconomic status, and people with addictions.

\section{Symptoms}

A mild TBI classifies when a head injury is accompanied by a loss of consciousness or dizziness in the time of injury, posttraumatic amnesia for less than one day, with Glasgow Coma scale (GCS) higher than 13, and negative results in neuroimaging diagnostics. The patients who suffer a mild TBI show emotional, cognitive, behavioral and physical symptoms, which occur separately or in combination and effect the individuals' functioning. The most common symptoms are a headache, dizziness, nausea, memory problems, fatigue, irritability, anxiety, insomnia, concentration difficulties, and light and sound sensitivity. ${ }^{15}$ Occurrence and perseverance of the cognitive symptoms after a mild TBI significantly influence the reintegration to the professional, academic and social activities. ${ }^{16}$ Despite the classification of the injury as "mild", the consequences can be persistent and persevering. The possible cognitive deficits that occur post-trauma express in form of attention, concentration, processing speed, memory, and executive functioning problems. ${ }^{17,18}$ In acute phase of the injury brain's metabolic activity changes and perfusions most commonly occur in the prefrontal cortex, reflecting on the impaired executive functioning. ${ }^{19}$ The expected cognitive symptoms rehabilitation takes from one week to 6 months whereas young athletes recover significantly faster. ${ }^{20}$ At this point it is not clear whether persisting cognitive symptoms come from the pathophysiological background of the injury or from other factors that could also influence cognitive functioning; for instance pain, fatigue, drugs, sleeping, pre-morbid personality factors, legal claims, psychological factors or emotional disturbances..$^{21,22}$ The cognitive sump-toms directly linked to the injury typically do not deteriorate with time. When the cognitive functioning problems persist after 3 months, a neuropsychological evaluation is recommended.

\section{Post-concussion syndrome}

A post-concussion syndrome develops after TBI regardless of the intensity of the injury. It is generally noticed that post-concussion symptoms along with a headache, dizziness and attention deficits result from neurophysiological influences. However, the persistent and long-term symptoms are harder to explain. The pre-injury 
and post-injury psychological factors significantly influence the persistence and exaggeration of the symptoms. Stressful life events, poor coping strategies, depression and individual fragility influence the psycho-genesis of the post-concussion syndrome, though concrete and objective evidence are limited. ${ }^{23-25}$

Numerous studies focused on potential demographic influences, preexisting variables, and premorbid factors that influence rehabilitation. The risk for slow rehabilitation is higher for persons with pre-injury problems such as addiction and lower psychosocial adaptation abilities. ${ }^{22}$ Long absenteeism and social withdrawal additionally effect personal devaluation and significantly alter emotional functioning. Some studies revealed that older (above 50) people are at higher risk for symptom pervasiveness than those affected at younger age. ${ }^{25-27}$ Many other factors can have an important role in recovery. For instance, individuals whose work require higher cognitive abilities are often slower to return to their pre-injury functioning level. Work environment that requires multi-tasking, fast decision making, priority assigning and high level of mental flexibility may pose difficulties for an individual who experienced a mild TBI Other post injury factors, which de-lay recovery, are a financial pressure, family members' indifference, chronic pain, looking for someone to blame for the accident, and resentment or anger in relation to accident. The rehabilitation can be additionally delayed because of the litigations or annuity tendencies. Some studies show that symptom pervasiveness is present equally in patients who underwent the litigations and those who have not, while other show significant positive relationship between pervasiveness and exaggeration of the symptoms and litigations. ${ }^{9,25,28}$

\section{Psychological Consequences}

\section{Higher cognitive function impairments}

Neuropsychological evaluation can help us diagnose cognitive and emotional disturbances and plan treatment and rehabilitation. Neuropsychological evaluation starts with the clinical interview, where we gain in-formation about basic health and psychological anamnesis, highest formal education level of the individual, presence of pre-morbid learning difficulties, existence of past head injuries and finishes with a detailed evaluation of a cognitive and emotional state.

The nature and seriousness of cognitive complaints are best to be evaluated with neuropsychological diagnostics by a qualified clinical psychologist with sub-specialization in neuropsychology. We gain important in-formation about patients' cognitive abilities that are otherwise difficult to detect. The patients themselves are usually not able to describe their problems as they have little or no insight, they can make mistakes at work but cannot understand the underlying cause. Family members are a better source of information in reporting patient's cognitive and behavioral changes.

Based on information gained with the clinical interview, patient's medical files, and medical diagnosis the neuropsychologist decides on cognitive and emotional aspects that need to be addressed and what test battery should be used. Most neuropsychologists use flexible test battery modified for every individual based on alleged impairments of cognitive, emotional and behavioral functions. ${ }^{29}$ The neuropsychological evaluation for patients who suffer a mild TBI is more complex and detailed than merely using a screening test. The use of the latter is appropriate when the patient is acutely ill or behaviorally problematic. However, in most cases the evaluation is wholesome and usually lasts 4 to 5 hours. The detailed inspection is more so needed when a patient complains about various cognitive functioning problems or when the clinical picture does not fit the nature of the injury. Veltman and colleagues ${ }^{30}$ evaluated 166 patients with mild TBI in the acute phase with a screening test and retested them in a later stage. The results showed that screening tests significantly accurately predicted the poorer recovery. The authors suggest that cognitive evaluation is useful in the early stages of the injury as it has a predictive power to categorize patients with a poorer expected outcome.

\section{Cognitive and emotional assessments usually included in the neuropsychological evaluation of a mild TBI are as following (3 I-35)}

Intelligence: It is very important to determine the intelligence level, which needs to be taken in consideration in further neuropsychological evaluation. Evaluating individual's intellect with a standardized test allows us to assess his pre-morbid functioning and evaluate whether his post-injury intellectual abilities are affected.

Attention: One of the key assessments in mild TBI is assessment of attention. Attention deficits can significantly influence the functioning of other cognitive abilities and can express as an overall dispersed and hindered functioning. Evaluation of the deficits in focused, selective, alternating, divided, and sustained attention can help us understand why a patient cannot follow the conversation, school lecture or perform in noisy and disturbing surroundings. The problems usually arise when attention needs to be alternated between different stimuli and tasks. For instance, a patient could burn the food while cooking because he cannot divide attention between cooking potatoes on a stove and preparing vegetables for a salad.

Memory: Evaluation of the memory normally includes assessment of abilities to sustain verbal and nonverbal material, assessment of a memory load, information retrieval or coding. The memory assessment helps us evaluate patient's memory storing abilities, the learning curve, whether cueing helps retrieve information from the memory or if there are difficulties with delayed recall. The problems often arise when retrieving new information from the long-term or working memory, where usually affected is also the ability to sustain information or manipulate information for speech formatting.

Higher cognitive functions (executive functions): The clinical assessment comprises of tests that are sensitive for frontal lobe functions. The tests include evaluation of the planning abilities, abstract thinking, concept formation, organization, reasoning, inhibition, thinking flexibility, initiation, and problem solving. The frontal lobe is also important in memory functioning through strategies that help retrieve information (e.g. semantic grouping).

Mental information processing: The information processing speed and speech formation is often delayed. The patient perceives the usual speech tempo as fast and often feels overwhelmed in a conversation.

Visuospatial abilities: Even though the specific problems with visual, spatial, or construction abilities are normally not expected in a mild TBI, the time expected for a task completion of the tests measuring those abilities often exceeds the norm. The results often show slower information processing abilities.

The emotional status: For objective evaluation of emotional disorders, the standardized tests for depression, anxiety, or other psychological states are used. It is important to take the potential presence of emotional disorder into consideration when assessing the cognitive deficits. 


\section{Behavioral and emotional changes}

Behavioral and emotional changes that follow a mild TBI can be very persistent, exhausting and can significantly alter the functioning of the patient and influence the dynamics of the family. ${ }^{36}$ The early symptoms of a mild TBI often include irritability, anxiety, emotional liability, depressive symptoms, and apathy. There is a significant prevalence of long lasting emotional disorders in patients with mild TBI, where most frequent are a major depressive disorder, anxiety disorders and irritability. The prevalence of depression in the mild TBI is $10-60 \%$ in the first year post injury ${ }^{37,38}$ and $17 \%$ even after $3-5$ years after injury. Depressive disorder that occurs after a traumatic injury often includes irritability and comorbid anxiety symptomatic. The latter include generalized anxiety disorder, panic attacks, phobias and posttraumatic stress disorder (PTSD). Psycho-logical problems can occur for the first time after the injury or reoccur as worsening of the pre-injury state. ${ }^{39}$

Regardless of whether the emotional disturbance has a direct causal connection to the brain injury, it is necessary to detect it in early stages and treat it as it can significantly influence the recovery of other symptoms of the post-concussion syndrome..$^{40,41}$

\section{Simulation detection}

The symptom simulation is an intentional reporting of false or exaggerated physical and/or psychological symptoms. The motives are usually externally motivated by avoiding military duties, avoiding work, gaining financial benefits, annuity, avoiding prosecution or other legal procedures, gaining drugs or merely to draw attention and compassion of others. ${ }^{42}$ Binder and colleagues ${ }^{42}$ reported a metaanalysis of 17 studies where investigation of 2353 patients showed that one of the significant risk factors for long-term impairment, symptom presence, and objective clinical signs after a mild TBI is involvement in the legal claims. Similar findings were reported in a Cassidy and colleagues $^{1}$ study of patients injured in motor vehicle accidents. The assessment of the simulation probability in the neuropsychological testing varies from $5-50 \%$ or more. ${ }^{43,44}$ The variance in simulation detection results from the evaluation procedure, nature of the referral, and definition or determination of either simulation, somatization, or conversion disorder. The differentiation can be difficult as the symptoms often overlap. In all of the above cases, we face the discrepancy between medical results and patient's complaint and the objective signs and subjective symptoms. ${ }^{45}$

\section{Clinical Psychological Psychotherapy}

Psychotherapy is crucial in treating mental disorder after a mild TBI. It includes support and problem solving strategies in addition to formal approach to psychotherapy. Cognitive Behavioral Therapy (CBT) is recommended for long lasting problems after a mild $\mathrm{TBI}^{46}$ and post-concussion syndrome in general, for treating PTSD and improving coping in stressful situations after the injury. Past experiences in CBT and patients with mild TBI generated models professionals can follow in order to treat most common symptoms associated with PCS including depression, anxiety, sleep problems, chronic fatigue, and chronic pain.

To create most successful treatment plan it is upon professionals' experiences and initial psychopathological and neuropsychological assessment to choose and adapt the model to patients' needs as the consequences of a mild TBI are subjective and unique to each patient. The careful consideration of techniques that CBT offers is often a first step toward successful treatment. The CBT includes a combination of strategies focused on symptoms to improve emotional state and change maladapted thought patterns and strategies focusing on inappropriate behaviors. CBT treatment involves psycho-education, cognitive restructuration, behavioral activation, teaching problem solving techniques and emotional regulation, and systematic desensitization of frightening situations in anxious states. Additionally, it includes strategies for pain management, insomnia, fatigue, and management of preoccupation with own health state.

As PCS has been repeatedly linked to the psychological origin which maintains a patient in a state where he or she feels his or her state is not improving. Using a CBT approach a professional tries to shape individual's attitude towards the symptoms. The first challenge a therapist encounters when patients with PCS is referred is to gain trust of a patient. Seeing therapist makes patients believe that professionals do not believe in the objective pain or disorder he or she is feeling or experiencing but with such referral imply that patients' problem is "imaginary", or originates in his or her "mind". Therefore, one of the initial and most important task is psychoeducation on the nature of mild TBI, and consequently of persisting PCS. When a patient understands the nature of disease, the role of vicious circle that maintains the symptoms and gains confidence on being able to control the symptoms in the future the therapy is most likely to succeed.

The proposed structure of a therapeutic approach is a Twelvesession formulation-led framework for CBT for persistent PCS (47). A first session, as already mentioned should be focused on psychoeducation on injury, recovery from injury, and the structure of the CBT. In the second session the focus is to identify he problems and to bring the wider perspective on the problems. One of the messages of CBT is that the thoughts, attitudes, and beliefs you hold have a big effect on the way you interpret the world around you and on how you feel. Emphasizing the thought feeling link is more so important in patients with PCS who see the sole origin of their symptoms as a consequence of the brain injury. In the third session the therapist and patient develop techniques that may ease the patient's problems, for example a patient who experiences chronic fatigue, learns ways to increase activation and social interaction. At the fourth session until the end of therapy the therapist and a patient work on concrete examples of patient's problems and ways to solve them. In CBT approach patients get to work outside of the therapeutic environment in form of homework and report success or struggles experienced at the time they applied problem solving strategy in their real life. Therapist helps patient to evaluate his performance and adapt the coping strategy to be more successful in a similar situation next time it occurs. One of the final, also trivial parts of the therapeutic process is the relapse prevention where the therapist starts preparing the patient for the end of the therapy process. That usually starts at the ninth session until the end of the treatment process. In relapse prevention it is important that patients feel confident in their self-management abilities and attributes the success to his own capabilities. It is important to maintain realistic expectations and prepare a patient for the relapse that is most likely to occur in the future. When a patient feels ready and equipped to cope with such situation it is most likely that relapse will be short-lasting.

A CBT approach is cooperative and problem-focused and has been shown to be effective in numerous conditions where specific symptoms need to be addressed. There is a large body of evidence and well established models in treating symptoms of anxiety, and depression, and also CBT is widely used in chronic fatigue, and chronic pain and other symptoms that are indicative for a PCS such as dizziness, headaches, and mood swings (47). 


\section{Case Report}

Described patient is a 38 year-old male (C.B.) at time of treatment, who was seen in consultation for a neuropsychological evaluation to identify the nature and extent of any neurocognitive deficits related to a possible mild traumatic brain injury secondary to attack on 12/31/2013 by several unknown aggressors. The patient suffered repeated beating to the head with fists and footwear. From the time of first hit until the time he managed to escape the attackers he does not have recollection of memory, however objective evidence of loss of consciousness is lacking. Upon arrival to the hospital several hours later, the GCS score was 15 . A contusion was noted at supraorbital right side and in the area of nose root. There was an evident nose bleed and blurred vision to the left eye. At the ER head x-ray did not confirm any skeletal damage, however he was sent for ophthalmologic consultation at the University Medical Centre. The consult was carried out the next day, on 1/1/2014 and revealed no pathology at the left eye area. The x-ray of the head, neck and right shoulder revealed no skeletal damage and neurological status has been reported as normal.

The patient returned to the Trauma department of the University Medical Centre after two days (1/3/2014) due to the nausea, vomiting, persistent headache and reported difficulties with hand motor coordination. At the upper limb examination a pronator drift to the left hand has been detected, an emergency head CT of the face and head has been performed. Head CT confirmed nose bone fracture with no intracranial post-trauma changes. He has been discharged from the hospital with the diagnosis concussion.

The patient complained of evident deficits following the attack and was included in neuropsychological treatment from August 2014 until March 2015. The following psychological consult revealed that patient reported no memory of the violent act. He is highly educated, has a master's degree in Economics, and was employed on a demanding leading position.

The psychological evaluation comprised of neuropsychological diagnostics, cognitive rehabilitation and cognitive behavioral therapy for coping with his current health state.

The neuropsychological screening tests included The Repeatable Battery for the Assessment of Neuropsychological Status (RBANS), Tower of London test (TOL), Category test, California verbal learning test, Comprehension mental tracking test, and Vienna test.

Neuropsychological screening test (RBANS) revealed a psychomotor slowing and clinically significant deficits in immediate memory recall (List Learning, 1 PR; Story Memory, 3 PR) and delayed memory recall (List Recognition, 0,01 PR; Story Recall, $0,01 \mathrm{PR})$. Clinically impaired were also executive functions (TOL). Attention assessment (Vienna test Schufried) revealed slowing in ability of response to basic visual stimuli regardless of the presence of the warning sound signal. The selective responsiveness to the stimuli was also slow with lowered inhibition ability (Reaction Time, 5 PR; Visual Pursuit test, 1 PR).

Recovery was evident after 7 months of treatment. In this time the second neuropsychological evaluation revealed that patient significantly improved on his cognitive skills that were after the treatment at the average and above-average level. Neuropsychological screening test (RBANS) revealed an improvement in psychomotor slowing and in immediate memory recall (List Learning, 84 PR; Story Memory, $93 \mathrm{PR}$ ) and delayed memory recall (List Recognition, 61 PR; Story Recall, 37 PR). Vienna test revealed a reaction time improvement (67 PR) and visual pursuit tests improvement to (41PR).
The cognitive rehabilitation focused on the deficits revealed by the neuropsychological testing and was including the computer based training (Rehacom) and paper-pencil exercises. The cognitive rehabilitation included tasks to improve memory, learning, mental processing speed, attention and executive functions. The patient received daily homework of exercises from cognitive rehabilitation textbook Brainwave translated to Slovenian.

CBT included psycho-education, cognitive restructuration, distraction technique, progressive muscle relaxation, relaxation breathing technique, behavior experiment. Cognitive behavioral therapy consisted of 8 sessions with one booster session. The emotional state evaluation revealed depression, the patient avoided social contacts, and free time activities he used to enjoy. Additional sessions of CBT were needed as the patient lost his job meanwhile rehabilitation process, because there was no objective evidence for a traumatic brain injury (CT and MRI showed no impairments) even though his inability to concentrate and poorer performance at work persisted. With CBT he accomplished cognitive restructuration, improved on his self-esteem, and was again active and social. Depressive symptoms were non present at the end of the treatment. At the end of rehabilitation and therapy program, his mood was euthymic with a normal affective range. He is now seeking a new job and continuing with his $\mathrm{PhD}$ thesis.

\section{Discussion}

Lack of objective clinical evidence at traumatic brain injury normally classifies the brain injury as "mild". The diagnosed patients are often overlooked despite their significant problems on cognitive and affective functioning. It is generally accepted that acute physical and cognitive symptoms have underlying neurophysiological causes whereas, long lasting symptoms of the mild TBI are often seen as a consequence of premorbid emotional and personality factors, current emotional state, stress, secondary benefits or the influence of the iatrogenic effects.

The cognitive and emotional problems of our patient persisted for six months after the blow to the head. He reported significant cognitive problems, especially in slowing of the mental processing, poorer attention, planning, and decision making. The disappointment about worse cognitive functioning generated the depressive symptoms with social avoidance. The patient felt misunderstood and often faced judgement from his peers and accusations about simulating symptoms. Even though there was clear evidence of cognitive slowing at the neuropsychological evaluation, he lost his job due to the lack of objective evidence. General presumption following concussion is that the difficulties should diminish after six months and when that did not happen the employee decided to terminate their collaboration. His workplace was intellectually highly demanding and not reaching the goals of the job posed additional stress to the patient as he himself noticed lowered abilities to perform at work post injury. The stress of losing his job additionally extended the recovery time as well as the fact that aggressors that attacked him were never identified and faced consequences for his injury.

It has been noted that an early psycho-education with elements of CBT significantly contributes to the recovery of persisting affective symptoms following mild TBI. ${ }^{48}$ In patients where symptoms persist 3 months after a traumatic brain injury the general recommendation applies to include them in a treatment program involving neuropsychological evaluation and diagnostics with elements of cognitive behavioral therapy. Left untreated, the risk for development of depressive symptoms is higher and can additionally prolong the recovery time. 
Even though single case studies report successful outcomes in treating post-concussion syndrome with CBT, the comprehensive and methodologically strong evidence is still lacking. The professionals dealing with such patients are in need of comprehensive treatment strategies and clinical guidelines to approach the patients with best outcome in mind. The recent systematic review found positive evidence for the effectiveness of treatment, however there was a small number (10) of studies with efficient research design with small number of participants and short follow-up. However, they conclude that based on the evidence thus far and the more strong body of evidence in effectiveness of the CBT in the chronic fatigue treatment which is parallel to PCS symptoms, one can cautiously conclude that CBT approach is beneficial for PCS treatment. ${ }^{49}$

Case studies are invaluable source of information for further development of proper methodology to evaluate the effectiveness of the cognitive behavioral therapy in mild traumatic brain injury and post-concussion syndrome.

\section{Acknowledgments}

None.

\section{Conflicts of Inetrset}

None.

\section{References}

1. Cassidy JD, Carroll L, Peloso P, et al. Incidence, riskfactors and prevention of mild traumatic brain injury: Results of the WHO Collaborating Centre Task Force on Mild Traumatic Brain Injury. $J$ Rehabil Med. 2004;(43 Suppl):28-60.

2. Raskin S, Mateer C. Neuropsychological management of Mild Traumatic Brain Injury. Oxford University Press, New York, USA. 2000.

3. Kurtzke JF, Jurland LT. The epidemiology of neurologic disease. In: Joynt RJ (Ed.), Clinical Neurology. JB Lippincott, Philadelphia, USA. 1993.

4. Bazarian JJ, McClung, Shah MN, et al. Mild traumatic brain injury in the United states, 1998-2000. Brain Inj. 2005;19(2):85-91.

5. Alves W, Macciocchi SN, Barth JT. Postconcussive symptoms after uncomplicated mild head injury. $J$ Head Trauma Rehabil. $1993 ; 8(3): 48-59$.

6. Levin HS, Mattis S, Ruff RM, et al. Neurobehavioral outcome following minor head injury: a three-center study. $J$ Neurosurg. 1987;66(2):234-243.

7. McCrea M, Kelly JP, Randolph C, et al. Immediate neurocognitive effects of concussion. Neurosurgery. 2002;50(5):1032-1040.

8. Alexander MP. Mild traumatic brain injury: pathophysiology, natural history and clinical management. Neurology. 1995;45(7):1253-1260.

9. Hugenholtz H, Stuss DT, Stetham LL, et al. How long does it take to recover from a mild concussion? Neurosurgery. 1988;22(5):853-858.

10. Caroll LJ, Cassidy JD, Peloso PM, et al. Systematic search and review procedures: results of the WHO Collaborating Centre Task Force on Mild Traumatic Brain Injury. J Rehabil Med. 2004;(43 Suppl):11-14.

11. Binder LM, Rohling ML, Larrabee GJ. A review of mild head trauma: Part I. Meta analytic review of neuropshychological studies. J Clin Exp Neuropsychol . 1997;19(3):421-431.

12. Kashluba S, Paniak C, Blake T, et al. A longitudinal, controlled study of patient complaints following treated mild traumatic brain injury. Arch Clin Neuropsychol . 2004;19(6):805-816.
13. Ponsford J, Willmott C, Rothwell A, et al. Factors influencing outcome following mild traumatic brain injury in adults. J Int Neuropsychol Soc. 2000;6(5):568-579.

14. Ruff RM, Camenzuli L, Mueller J. Miserable minority: Emotional risk factors that influence the outcome of a mild traumatic brain injury. Brain Inj. 1996;10(8):551-565.

15. Dikmen S, Machamer J, Temkin N. Mild head injury: Facts and artifacts. J Clin Exp Neuropsychol . 2001;23(6):729-738.

16. Vanderploeg RD, Curtiss G, Luis CA, et al. Long-term morbidities following self-reported mild traumatic brain injury. $J$ Clin Exp Neuropsychol . 2007;29(6):585-598.

17. Frenchmen KA, Fox AM, Mayberry MT. Neuropsychological studies of mild traumatic brain injury: A meta-analytic review of research since 1995. J Clin Exp Neuropsychol . 2005;27(3):334-351.

18. Silver JM, McAllister TW, Arciniegas DB. Depression and cognitive complaints following mild traumatic brain injury. Am J Psychiatry. 2009;166(6):653-661.

19. Metting Z, Rodiger L, Stewart R, et al. Perfusion computed tomography in the acute phase of mild head injury: region dysfunction and prognostic value. Ann Neurol . 2009;66(6):809-816.

20. Iverson GL, Lange RT, Brooks BL, et al. "Good Old Days" bias following mild traumatic brain injury. Clin Neuropsychol. 2010;24(1):17-37.

21. Bigler ED. Neuropsychology and clinical neuroscience of persistent post-concussion syndrome. J Int Neuropsychol Soc. 2008;14(1):1-22.

22. Wood RL. Understanding the 'miserable minority': A diasthesisstress paradigm for post-concussional syndrome. Brain Inj. 2004;18(11):1135-1153.

23. Bohnen N, Jolles J, Twijnstra A. Neuropsychological deficits in a patient with persistent symptoms six months after mild head injury. Neurosurgery. 1992;30(5):692-696.

24. Cicerone KD. Psychotherapy after mild traumatic brain injury: Relation to the nature and severity of subjective complaints. Journal of Head Trauma Rehabilitation. 1991;6(4):30-43.

25. Fenton G, McClelland R, Montgomery A, et al. The postconcussional syndrome: Social antecedents and psychological sequelae. $\mathrm{Br} J$ Psychiatry. 1993;162:493-497.

26. Radanov BP, Stefano GD, Schnidrig A, et al. Role of psychosocial stress in recovery from common whiplash. The Lancet. 1991;338(8769):712-715.

27. Williams DH, Levin HS, Eisenberg HM. Mild head injury classification. Neurosurgery. 1990;27(3):422-428.

28. Gfeller JD, Chibnall JT, Duckro PN. Postconcussion symptoms and cognitive functioning in posttraumatic headache patients. Headache. 1994;34(9):503-507.

29. Rabin LA, Barr WB, Burton LA. Assessment practices of clinical neuropsychologists in the United States and Canada: A survey of INS, NAN, and APA Division 40 members. Arch Clin Neuropsychol . 2005;20(1):33-65.

30. Veltman RH, VanDongen S, Jones S, et al. Cognitive screening in mild brain injury. J Neurosci Nurs. 1993;25(6):367-371.

31. Vanderploeg RD, Curtiss G, Belanger HG. Long-term neuropsychological outcomes following mild traumatic brain injury. J Int Neuropsychol Soc. 2005;11(3):228-236

32. Mathias JL, Bell JA, Bigler ED. Neuropsychological and information processing deficits following mild traumatic brain injury. $J$ Int Neuropsychol Soc. 2004;10(2):286-297.

33. Voller B, Benke R, Benedettos K, et al. Neuropsychological, MRI, and EEG findings after very mild traumatic brain injury. Brain Inj.1999;13(10):821-827. 
34. Carroll L, Cassidy J, Peloso P, et al. Prognosis for mild traumatic brain injury: Results of the WHO Collaborating Centre Task Force on Mild Traumatic Brain Injury. J Rehabil Med. 2004;43(suppl):84-105.

35. Uzzell B. Mild head injury: Much ado about something. In: Varney N \& Roberts R (Eds). The Evaluation and Treatment of Mild Traumatic Brain Injury. Lawerence Erlbaum, New Jersey, USA. 1999. p.1-13.

36. Lezak MD. Relationships between personality disorders, social disturbances and physical disability following traumatic brain injury. Journal of Head Trauma Rehabilitation. 1987;2(1):57-69.

37. Dikmen SS, Bombardier CH, Machamer JE, et al. Natural history of depression in traumatic brain injury. Arch Phys Med Rehabil . 2004;85(9):1457-1464.

38. O’Donnell ML, Creamer M, Pattison P, et al. Psychiatric morbidity following injury. Am J Psychiatry. 2004;161(3):507-514.

39. Whelan-Goodinson R, Ponsford JL, Schönberger M, et al. Predictors of psychiatric disorders following traumatic brain injury. $J$ Head Trauma Rehabil. 2010;25(5): 320-329.

40. Fann JR, Uomoto JM, Katon WJ. Cognitive improvement with treatment of depression following mild traumatic brain injury. Psychosomatics. 2001;42(1):48-54.

41. Rapoport MJ, McCullagh S, Streiner D, et al. The clinical significance of major depression following mild traumatic brain injury. Psychosomatics. 2003;44(1):31-37.

42. Binder LM, Rohling ML. Money matters: a meta analytic rewiev of the effects of financial incentives on recovery after closed head injury. Am J Psychiatry. 1996;153(1):7-10.
43. Binder LM. Assessment of malingering after mild head trauma with the Portland Digit Recognition Test. J Clin Exp Neuropsychol. 1993;15(2):170-182.

44. Kay T. Neuropsychological diagnosis: disentangling the multiple determinants of functional disability after mild traumatic brain injury. In: Horn L \& Zasler N (Eds.), Rehabilitation of Post-Concussive Disorders. Henley and Belfus, Philadelphia, USA. 1992. p.109-127.

45. Resnick PJ. Malingering of posttraumatic disorders. In: R. Rogers (Ed.), Clinical Assessment of Malingering and Deception. Guilford Press, New York, USA. 1988. p.3453.

46. Tiersky LA, Anselmi V, Johnston MV, et al. A trial of neuropsychologic rehabilitation in mild-spectrum traumatic brain injury. Arch Phys Med Rehabil . 2005;86(8):1565-1574.

47. Potter S, Brown RG. Cognitive behavioural therapy and persistent postconcussional symptoms: Integrating conceptual issues and practical aspects in treatment. Neuropsychol Rehabil. 2012;22(1):1-25.

48. Borg J, Holm L, Cassidy JD, et al. Diagnostic procedures in mild traumatic brain injury: results of the WHO collaborating centre task force on mild traumatic brain injury. J Rehabil Med. 2004; (43 Suppl):61-75.

49. Sayegh AA, Sandford D, Carson AJ. Psychological approaches to treatment of postconcussion syndrome: a systematic review. $J$ Neurol Neurosurg Psychiatry. 2010; 81(10):1128-1134. 